\title{
A DEFINIÇÃO DO ZERO EM FREGE: 0 COMPROMISSO DO PLATONISMO FREGEANO COM A “ETERNIDADE” DE BOLZANO.
}

\author{
Walter Gomide \\ Universidade Federal do Mato Grosso
}

\begin{abstract}
In this article I try to show how Frege's Platonism is related to the notion of Bolzano's eternity. Understood as the complete range of time-variation, Bolzano's eternity gives us a interesting instrument to conceive what is an eternal object: an object is said eternal if it is unchangeable through the flow of time. In that sense, Frege's definition of zero uses tacitly such notion as long as zero is defined as the "number that belongs to a concept" whose extension is unchangeable in each possible word (such worlds conceived as instants situated at Bolzalno's Eternity). That's why we can infer that Platonism, on Frege's view, may assume that abstract objects are not necessarily outside space and time, but are eternals, in the bolzanian sense.
\end{abstract}

Keywords: Frege, Bolzano, concept, extension of concept, eternity.

Resumo: Neste artigo, tento mostrar como o Platonismo de Frege relaciona-se muito intimamente com a noção de eternidade de Bolzano. Compreendida como o domínio total de variação do tempo, a eternidade de Bolzano nos oferece um interessante instrumento para estipular o que é eterno: um objeto é eterno se é imutável em relação ao fluxo do tempo. Desta forma, a definição do zero proposta por Frege faz uso tácito de tal noção, na medida em que o zero é definido como "o número que pertence a um conceito" cuja extensão é imutável em qualquer mundo possível (tais mundos sendo concebidos como instantes na 'Eternidade' de Bolzano). Por conta disto, podemos inferir que o platonismo, sob a ótica de Frege, pode assumir que os objetos abstratos não estão necessariamente fora do tempo e do espaço, mas são eternos, no sentido bolzaniano.

Palavras-chave: Frege, Bolzano, conceito, extensão de conceito, eternidade. 


\section{Introdução: platonismo e atemporalidade em Frege}

Não há dúvida de que um dos postulados mais bem assentados do platonismo é o que afirma que os objetos matemáticos, em especial os números, são atemporais ou, dito de outra forma, supra sensíveis. Na base deste pressuposto há o princípio de que o tempo é um elemento exógeno do mundo dos objetos matemáticos ${ }^{1}$. Em qualquer ontologia que se alinhe com a abordagem platonista, percebe-se a preocupação de eliminar a temporalidade do edifício ideal por meio do qual o mundo matemático se estrutura. Geralmente, este edifício abstrato é constituído de objetos, propriedades destes objetos, as propriedades das propriedades destes objetos, etc, sem que o tempo aí seja inserido como um elemento estruturante desta arquitetura.

Em Frege, por exemplo, o viés platonista quanto aos números talvez seja um dos mais recrudescidos de que se tenha notícia e, por conta disto, a expurgação do tempo (e do espaço) da esfera dos objetos matemáticos - no caso dos números, compreendidos por Frege como objetos estritamente lógicos - faz-se da maneira convicta. Pois, para Frege, ao contrário do que é afirmado por Kant, os objetos não são dados a nós necessariamente passando pela sensibilidade, isto é, pelas formas a priori do espaço e do tempo, princípios constitutivos da experiência, mas admitem ser acessados de forma completamente independente das intuições espaço-temporais. Segundo Frege:

Devo protestar contra a generalidade do [dictum] de Kant: sem a sensibilidade, nenhum objeto nos seria dado ${ }^{2}$. O Zero e

\footnotetext{
1 De fato, a tese de que o tempo não participa diretamente dos objetos matemáticos deve se restringir ao platonismo, uma vez que o intuicionismo, por exemplo, apresenta justamente um princípio oposto. Segundo o intuicionismo, os objetos matemáticos são construções temporais, devendo ser intrinsecamente associados a estas construções (ver BROUWER, [1949]).

20 dictum kantiano referido por Frege é o seguinte: "Se chamarmos sensibilidade à receptividade do nosso espírito em receber representações na medida em que de algum modo é afetado, o entendimento é, em contrapartida, a capacidade de produzir representações ou a espontaneidade do conhecimento. Pelas condições da nossa natureza a intuição nunca pode ser senão sensível, isto é, contém apenas a maneira pela qual somos afetados pelos objetos, ao passo que o entendimento é a capacidade de pensar o objeto da intuição sensível. Nenhuma destas qualidades tem primazia sobre a outra. Sem a sensibilidade, nenhum objeto nos seria dado; sem 0 entendimento, nenhum seria pensado. Pensamentos sem conteúdo são vazios; intuições sem conceitos são cegas" (KANT, [1781], A 51/B 75).
} 
o Um são objetos que não podem ser-nos dados pela sensação. E mesmo aqueles que sustentam que os pequenos números são passíveis de intuição, devem concordar que números maiores $[\ldots]$ não podem ser dados pela intuição (FREGE, [1883], §89).

O que Frege quer dizer na passagem acima é claro: para termos acesso aos números, não necessitamos da sensibilidade, isto é, não precisamos representá-los como objetos dados no espaço e no tempo. De fato, na ontologia fregeana os números são objetos definidos inteiramente dentro da lógica, ciência geral que nos dá as leis do pensamento ${ }^{3}$ e, como tais, descartam o espaço e o tempo como elementos constitutivos. O zero, nos Fundamentos da Aritmética (Grundlagen der Arithmetik) de 1884, por exemplo, é definido da forma seguinte ${ }^{4}$ :

Uma vez que nada cai sob o conceito "não idêntico a si mesmo", defino o zero como se segue:

0 é o numero que pertence ao conceito "não idêntico a si mesmo" (idem, ibidem, §74)

Expresso no simbolismo do cálculo lambda, a definição de Frege para o 0 é a seguinte:

(1) $0 \equiv_{\mathrm{df}}\{F / F \approx[\lambda x(\mathrm{x} \neq x)]\}$.

Em outras palavras, o número 0 consiste no conjunto - ou, grosso modo, a extensão de conceito, segundo terminologia fregeana, posto que a

\footnotetext{
${ }^{3}$ Afirmar que, em Frege, a lógica se identifica com as leis do pensamento é perfeitamente aceitável, pelo menos em relação à fase inicial do logicismo fregeano, como se depreende do título do livro que é considerado como o marco zero do programa logicista: Conceitografia, uma lingugagem formal do pensamento puro, modelada de forma aritmética (Begriffsschriftt, einer arithnetischen nachgebildete Formelsprache des reinen Denken) (ver BYNUM, [1972]).

${ }^{4} \mathrm{Na}$ definição que se segue, Frege utiliza-se da expressão "o número que pertence ao conceito $F$ ". Representando tal expressão por $F^{n}$, a definição da mesma é a seguinte FREGE, [1884], §73: $F^{n} \equiv_{\text {df }}\{G / G \approx F\}$.

$\mathrm{Na}$ definição acima, o símbolo " $\approx$ " significa a relação de segunda ordem "ser equinumérico": dois conceitos são equinuméricos se, e somente se, as suas respectivas extensões estão em correspondência bijetiva.
} 
extensão de um conceito $F$ consiste de todos os objetos que caem sob o conceito $F$, isto é, satisfazem a função proposicional $F x$ - composto de todos os conceitos que são equinuméricos ao conceito $[\lambda \mathrm{x}(\mathrm{x} \neq x)]$ - leia-se: "ser um $x$, tal que $(x \neq x)$ "-, sendo a relação de equinumericidade " $\approx$ " definida por completo somente com noções lógicas ${ }^{5}$. Nesta definição do zero, segundo a concepção de Frege, só entram elementos lógicos e, portanto, exclui-se de imediato que o zero - ou qualquer outro número natural $^{6}$ - possa ter como partícipe de sua natureza fatos ou objetos empíricos, ou de caráter subjetivo ou psicológico, posto que a lógica é radicalmente um domínio de coisas abstratas, como "conceito", "função", "propriedade", etc; e, por conseguinte, elimina-se dos números qualquer participação essencial do tempo como elemento constitutivo.

Analisando-se mais detidamente a definição que Frege apresenta do zero, vemos que nela aparece o conceito $[\lambda x(x \neq x)]$. A extensão do conceito "ser equinumérico ao conceito $[\lambda x(x \neq x)]$ é, essencialmente, o objeto lógico zero. Isto significa que os conceitos que compõem o zero são aqueles sob os quais nenhum objeto cai - ou que determinam extensão vazia -, uma vez que o conceito $[\lambda x(x \neq x)]$ é autocontraditório e, em uma ontologia normal, não pode haver um objeto que não seja idêntico a ele mesmo.

\footnotetext{
${ }^{5} \mathrm{~A}$ redução do conceito de equinumericidade à lógica passa necessariamente pela tradução de bijeção para o aparato lógico. Frege considera que isto é feito a contento de forma seguinte:

[Para que uma correspondência $\phi$ seja considerada bijetiva], ela deve satisfazer as duas condições seguintes:

i. Se $d$ está na relação $\phi$ com $a$, e se $d$ está na relação $\phi$ com e, então temos que, quaisquer que sejam $d$, a e e, a é o mesmo que $e$.

ii. Se $d$ está na relação $\phi$ com $a$, e se $b$ está na relação $\phi$ com $a$, então, quaisquer que sejam $d, b$ e $a$, então $d$ é o mesmo que $b$.

[Estas duas condições] reduzem a correlação [bijetiva] a relações puramente lógicas (idem, ibidem, §72).

${ }^{6}$ Os demais números naturais são logicamente definidos da forma seguinte:

$1 \equiv_{\mathrm{df}}\{F / \quad F \approx[\lambda x(x=0)]\}$.

$2 \equiv \mathrm{df}\{F / F \approx[\lambda x(x=0 \quad \vee x=1)]\}$.

$\mathrm{n} \equiv \mathrm{df}\{F / F \approx[\lambda x(x=0 \vee x=1 \vee \ldots \vee x=n-1)]\}$.
}

As definições dos números naturais surgem da proposição lógica "existe um conceito $F$ e um objeto $x$ caindo sob ele, tal que o número que pertence ao conceito $F$ é $n$, e o número que pertence ao conceito 'caindo sob $F$, mas diferente de $x$ ' é $m$ ", a qual Frege considera como o fundamento lógico da relação de sucessão imediata, na série dos naturais, entre $m$ e $n$. Com isto, Frege pretende que a equação $n=m+$ 1 seja completamente traduzida para conceitos e relações lógicas (idem, ibidem, §72). 
Mas não só o conceito $[\lambda x(x \neq x)]$ poderia ter sido usado para definir o zero. Segundo o próprio Frege, qualquer conceito que encerre em si uma contradição pode ser usado para definir o zero (idem, ibidem, §74); o que se exige de um conceito para que ele sirva como base à definição do zero, além de um claro critério da aplicabilidade - o seu caráter sortal (idem, ibidem, §74), é que a sua extensão seja vazia. E, de fato, qualquer conceito autocontraditório, na qualidade de uma função proposicional, sempre tomará valor falso para os seus argumentos, quaisquer que estes sejam, o que acarreta que a extensão destes conceitos - isto é, a classe ou conjunto dos objetos que caem sob os mesmos - seja vazia.

Daí, podemos inferir que, na definição do zero, subjaz o seguinte condicional:

$$
\text { (F1) } \forall G(0=\{F / F \approx G\} \rightarrow \varepsilon G=\varnothing)
$$

O condicional (F1) nos diz que, para qualquer conceito $G$, se este é usado para definir o zero - uma definição teórica, não nominal, o que acarreta a substituição do símbolo de definição por um de identidade como o número que pertence a tal conceito, então a extensão de tal conceito (simbolizada por " $\varepsilon G$ ") - isto é, o domínio de objetos que caem sob tal conceito - é vazia.

Parece ser um tanto quanto óbvio que o conceito usado na base da apresentação lógica do zero como o número que pertence a tal conceito tenha de ter uma extensão vazia; e é esta obviedade lógica que o condicional acima expressa. No entanto, se (F1) é evidente, o condicional seguinte parece não ter o mesmo grau de evidência:

$$
\text { (F2) } \forall \mathrm{G}(\varepsilon G=\varnothing \rightarrow 0=\{F / F \approx G\}) \text {. }
$$

Em (F2), o que é implicado em (F1) passa a implicar: afirma-se aqui que, se a extensão de um conceito é vazia, então o zero é definido como o número que pertence a tal conceito. Mas tomemos um conceito que contigentemente tenha extensão vazia. Para tal conceito, é possível postularmos um instante de tempo, diferente do presente, ou algum mundo possível para os quais a sua extensão não seja vazia - é o caso, por exemplo, do conceito "ser exemplar vivo do Tyrannosaurus Rex, ou ser cavalo alado, ou homem com idade maior ou igual a 170 anos": não é nada impossível 
(ou mesmo improvável), seja em alguma linha de tempo que tenha por origem o instante atual, qualquer que este seja, ou em algum mundo diferente daquele que vivenciamos como realidade, a existência de objetos que caiam sob tal conceito. Para tais conceitos cuja extensão é vazia por conta de uma contingência, (F2) não se aplica: o que se espera do zero é que ele seja o número que pertence a um conceito de extensão nula em qualquer instante de tempo passado, presente ou futuro, e em qualquer mundo possível; um zero que permita ser o número de um conceito sob o qual algo caia, certamente é inadequado ao propósito fregeano de apresentar uma fundamentação lógica dos números como representativos de cardinalidades: se a resposta à pergunta "quantos F's há?" for "nenhum”, espera-se que o zero seja o número pertencente a $F$, independentemente de contextos espaço-temporais, ou de mundos possíveis. Por conseguinte, os conceitos que são aptos a ter o zero como o número que lhes pertence devem ter extensão nula em qualquer instante de tempo, e em qualquer mundo possível. Desta forma, subjacente à definição do zero em Frege, reside o seguinte princípio:

$$
\text { (F3) } \forall G(\forall w \forall t \varepsilon G(w, t)=\varnothing \rightarrow 0=\{F / F \approx G\})
$$

Em (F3), a expressão " $\varepsilon G(w, t)$ " significa uma extensão de conceito que é função tanto do tempo, quanto de mundos possíveis - incluso dentre estes, obviamente, o mundo real. Em um primeiro momento, considerar que "extensões de conceito", objetos lógicos por excelência, possam variar no tempo tal qual um objeto físico, soa um contra-senso tratando-se do logicismo fregeano. Mas o próprio Frege, em seus Grundlagen, admite que os conceitos - ou, melhor dizendo, as suas extensões - carreiam consigo algo fluido, mutável no tempo. Eis o que nos diz Frege:

[O] conceito "habitante da Alemanha" contém uma referência ao tempo como um elemento variável, ou, dito de forma matemática, é uma função do tempo. [...] Portanto, no conceito em si mesmo [isto é, em sua extensão] ${ }^{7}$, também há

\footnotetext{
7 Em uma nota de rodapé dos Grundlagen, Frege assume que as expressões "extensão de conceito" e "conceito" serão usadas como sinônimos. Conforme nos diz Frege: "Creio que por "extensão de conceito" podemos simplesmente escrever "conceito". Mas isto estaria sujeito a duas objeções:
} 
alguma fluidez [So ist also in dem Begriffe selbst schon etwas Fliessendes] (idem, ibidem, §46).

1) As extensões de conceito como objetos variáveis no tempo e a "eternidade" de Bolzano.

Na passagem acima, Frege deixa claro que as extensões de conceito (vide nota 7) podem variar no tempo. Como tais, as extensões de conceitos situam-se em pé de igualdade com os objetos ou grandezas físicos, no sentido que, como estes, as extensões podem mudar com o tempo ou permanecer constantes em relação ao fluxo temporal.

Estas considerações trazem à luz a análise do matemático e filósofo (e também eclesiástico) de origem tcheca Bernard Bolzano (1781-1842) sobre o papel do tempo na constituição dos objetos. Em seu Ensaio para uma Fundamentação Objetiva da teoria das três Dimensões do Espaço (Versuch einer objectiven Begründung der Lehre von den drei Dimensionen des Raumes), de 1842, Bolzano nos diz:

[O] tempo no qual uma coisa se encontra, embora não pertença propriamente às propriedades [da mesma], certamente é uma de suas determinações [...] (BOLZANO, [1842], §2.5) ${ }^{8}$.

Bolzano afirma que o tempo não faz parte das propriedades (Beschaffenheiten) das coisas, embora seja uma de suas determinações. De fato, no mesmo ensaio, é dito que "o tempo não uma propriedade inerente

1) isto contradiz minha tese anterior de que os números individuais são objetos, como indicado pelo uso do artigo definido em expressões como "o número dois", e pela impossibilidade de falar de uns, dois, etc, no plural, como também pelo fato de que o número constitui somente um elemento no predicado de uma asserção numérica;

2) conceitos podem ter extensões idênticas sem com isto coincidirem entre si.

Estou convencido [...] de que ambas objeções podem ser feitas; mas fazê-las iria nos levar muito além dos propósitos presentes [aber das möchte hier zu weit führen]. Tomo por posto que o que é a extensão de um conceito seja conhecido. (idem, ibidem, nota 1, §69).

80 trecho original, em alemão, é como se segue: "[Die] Zeit, in der sich ein Ding befindet, obgleich sie nicht zu den Beschaffenheiten desselben gehört, doch gewiss eine seiner Bestimmungen ist [...] "(BOLZANO, [1842], §2.2). 
das coisas, nas quais ele possa ser encontrado"9 (idem, ibidem, §2.2). Não sendo uma propriedade das coisas, o tempo se insere na constituição do mundo como uma das determinações que as coisas ou os objetos podem possuir. Em 1853, com os seus Paradoxos do Infinito (Paradoxien des Unendlichen), Bolzano deixa claro em que sentido o tempo é uma das determinações das coisas: o tempo é a condição pela qual os objetos mudam ou variam. Segundo Bolzano:

[O] tempo, para mim, não é qualquer coisa variável, mas certamente [isto em que toda variação tem lugar ]. Quando se diz o contrário - como no provérbio: o tempo muda -, entende-se por tempo as coisas que nele se encontram e seus estados [...]. O tempo, em si mesmo, é a determinação inerente a toda substância variável, ou (o que dá na mesma) dependente [do tempo] (BOLZANO, [1853], §39)*.

O tempo, na concepção bolzaniana, é o que possibilita a mudança ou variação das coisas ou dos objetos. Mais precisamente, qualquer mudança ou processo se faz no tempo; sem este, a condição de possibilidade da mudança, a noção de variação é desprovida de sentido. Também no Ensaio, encontramos a seguinte passagem, muito ilustrativa da posição de Bolzano relativa ao tempo:

Finalmente, investiguemos mais precisamente que tipo de determinação é esta que pensamos para um objeto, quando consideramos o mesmo como situado neste ou naquele instante de tempo: então, dificilmente irá nos escapar que as determinações do tempo têm a propriedade de que elas se encontram somente nas coisas que são reais, devendo ser encontradas nestas, tomadas em sua totalidade - com exceção da [...] perfeita substância da divindade. Para cada coisa real, se ela depende de algo, associa-se [a mesma] a

\footnotetext{
9 "Die Zeit is nicht auch keine eigentliche Beschaffenheit der Dinge, die sich in ihr befinden" (BOLZANO, [1842], §2.2).

* Tradução feita pelo autor do artigo, diretamente da versão em francês Les Paradoxes de L'Infini, introduction et traduction de Hourya Sinaceur. Éditions du Seuil, Paris, [1993].
} 
determinação do tempo. Além disso, não nos pode passar desapercebido que todas estas [...] coisas reais são variáveis, sendo somente no tempo [que as mesmas podem se alterar] (BOLZANO, [1842], §2.6). ${ }^{10}$

Mais uma vez, Bolzano enfatiza que o tempo é a condição de possibilidade para que as coisas reais [Wirklichen] se alterem. No caso das extensões de conceito, se estas variam, então são objetos no tempo; é o tempo que permite que falemos de tais extensões como, por exemplo, agora sendo vazias, mas posteriormente não mais o sendo. Por conseguinte, uma extensão de conceito constantemente vazia é um caso de um objeto que se preserva sempre o mesmo no fluxo do tempo; o conceito ao qual o zero pertence tem sua extensão como exemplar desta propriedade de ser permanentemente vazia em relação a todos os instantes de tempo.

Segundo Bolzano, a totalidade destes instantes de tempo é a eternidade e, na concepção bolzaniana, os objetos que são sempre o mesmo no decorrer destes instantes - como é o caso "da perfeita substância da divindade" [der allvolkommenen Substanz der Gottheit] - são chamados de eternos. Considerando que a noção de instante é de fundamental importância para a apresentação do princípio de não-contradição, Bolzano introduz o tempo inteiro ou a eternidade da forma seguinte:

[A] representação do tempo deve se ajustar a da substância, a fim de permitir que, sendo dado um par qualquer de propriedades contraditórias $b$ e não-b, possamos atribuir uma em detrimento da outra. Mais precisamente, a determinação da qual falo é uma única parte de tempo, um ponto no tempo, um instante no qual nós devemos representar a substância $x$ para a qual nós queremos atribuir com certeza uma das duas propriedades de qualquer par de

\footnotetext{
10 "Untersuchen wir endlich genauer, was für eine Bestimmung es sei, welche wir uns an einem Gegenstande denken, wenn wir denselben als befindlich in diesem oder jenem Zeitpuncte denken; so wird uns kaum entgehen, dass die Bestimmungen der Zeit das Eigne, dass sie sich nur an Dingen, die etwas Wirkliches sind, befinden, an diesen aber auch durchaus- - mit Ausnahme der [...] allvollkommenen Substanz der Gottheit - anzutreffen sein müssen. An jedem Wirklichen, wenn es ein Abhängiges ist, haftet die Zeitbestimmung. Es kann auch nicht entgehen, dass alle diese bedingten Wirklichen verändlich sind, und dass es eben nur die Zeit sei, in [welcher sie sich verändern können]" (BOLZANO, [1842], §2.6)
} 
propriedades contraditórias $b$ e não-b; deste modo, diremos exatamente [desta maneira]: no instante $t, x$ ou bem tem a propriedade $b$, ou bem tem a propriedade não- $b$. Se considero que tal definição de instante é correta, posso então dizer claramente o que é o tempo propriamente, o tempo inteiro ou a eternidade: é o todo ao qual pertencem, enquanto partes, todos estes instantes [...] A conseqüência destas definições é a ausência de diferença entre o tempo e a eternidade, uma vez que se entende por tempo não um tempo limitado, finito [...], mas o tempo como um todo, ilimitado nos dois sentidos.

Entretanto, Deus e as coisas criadas estão no tempo de maneiras estritamente diferentes. As criaturas, em efeito, estão no tempo na medida em que elas mudam, enquanto Deus permanece sempre o mesmo, invariavelmente idêntico a si. Esta é a razão pela qual chamamos Deus de eterno, enquanto as criaturas são chamadas de temporais (BOLZANO, [1853], §39).

\section{A extensão do conceito "ser diferente de si mesmo" como necessariamente eterna e vazia}

Em relação à eternidade de Bolzano e aos conceitos, surge então a seguinte questão: há algum conceito que defina uma extensão eterna e vazia? Em outras palavras, há algum conceito que instancie existencialmente a seguinte expressão:

$$
\text { (F5) } \exists \chi \forall \boldsymbol{t} \varepsilon \chi(\boldsymbol{t})=\varnothing \text {. }
$$

Em (F5), a quantificação universal para os instantes de tempo pressupõe a eternidade de Bolzano: trata-se aqui de considerar que, para qualquer instante $\boldsymbol{t}$ (sendo este presente, passado ou futuro), há um conceito cuja extensão é vazia em relação a $\boldsymbol{t}$; postula-se aqui um conceito cuja extensão seja nula e bolzaniamante eterna. Mas que conceitos satisfazem tal critério? Para tanto, Frege aposta nos conceitos autocontraditórios, em especial o que postula que há objetos que não são iguais a si mesmos. Um 
tal conceito, evidentemente, terá necessariamente extensão sempre vazia, posto que consiste em uma negação explícita de um princípio ontológico - o de identidade. De fato, está subjacente na definição do zero como o número que pertence ao conceito "ser não idêntico a si mesmo" o seguinte $\operatorname{argumento}^{11}$, no qual fica clara a dependência de tal definição a uma proposição em que a temporalidade total - ou a eternidade, segundo Bolzano - está presente:

1) $\forall G(\forall \boldsymbol{t} \varepsilon G(\boldsymbol{t})=\varnothing \rightarrow 0=\{F / F \approx G\})$ (F6) Hipótese

2) $\exists \chi \forall t \varepsilon \chi(t)=\varnothing(F 5)$

3) $\forall \boldsymbol{t} \varepsilon[\lambda x(x \neq x)](\boldsymbol{t})=\varnothing$ (2) Inst. Exist (Hipótese)

4) $\forall \boldsymbol{t} \varepsilon[\lambda x(x \neq x)](t)=\varnothing \rightarrow 0=\{F / F \approx[\lambda x(x \neq x)]\})$ (1) Inst. Universal

5) $0=\{F / F \approx[\lambda x(x \neq x)]\}$ (3), (4) Modus Ponens.

Pelo argumento acima, a apresentação do zero como o número que pertence a $[\lambda x(x \neq x)]$ justifica-se na medida em que se pressupõe que a extensão de tal conceito, para todos os instantes de tempo, é nula. Se tomarmos tal argumento como a fundamentação lógica da identidade entre “0” e “ $\{F / F \approx[\lambda x(x \neq x)]$ ”, então podemos concluir que, embora o zero seja definido somente com noções lógicas, a justificação para esta definição pressupõe o tempo tomado como a totalidade dos instantes. Por conseguinte, o caráter abstrato do zero - caráter este que gera a crítica anteriormente vista que Frege faz a Kant - se põe ancorado na tese de que a extensão do conceito "ser diferente de si mesmo" é sempre nula, em qualquer instante considerado, sendo passado, presente ou futuro.

Poder-se-ia criticar tal argumento insistindo-se na tese de que, assim como uma extensão de conceito eternamente nula implica que tal conceito está apto a ser o conceito arquetípico ao qual o zero pertence, a

\footnotetext{
$11 \mathrm{Em}$ todos os argumentos utilizados nestes artigo, pressupõe-se uma lógica de segunda ordem bem intuitiva, na qual o comportamento dos quantificadores existencial e universal, uma vez quantificando conceitos, seja o mais similar possível ao uso que destes fazemos na lógica de primeira ordem, ao quantificarmos para indivíduos. As regras de inferência de uma lógica deste tipo podem ser encontrada em ZALTA, [1981], pp 30-32). Além de tais regras de inferência, tal lógica conta com o problemático axioma $\mathrm{V}$ de Frege, o qual associa conceitos a suas respectivas extensões, a saber (ver FREGE, [1893]):
}

(V) $(\varepsilon G=\varepsilon F) \leftrightarrow(\forall x)(G X \leftrightarrow F x)$ 
recíproca também é verdadeira: se o zero pertence a um conceito autocontraditório - como é o caso de $[\lambda x(x \neq x)]$-, então a extensão de tal conceito é nula em quaisquer instantes de tempo. Formalmente, tal argumento é apresentado a partir de uma versão mais forte de (F1), a saber:

$$
\text { (F1') } \forall G\left[\left(G^{*} \wedge 0=\{F / F \approx G\}\right) \rightarrow \forall t \varepsilon G(t)=\varnothing\right]
$$

Em (F1'), “ $G *$ ”' significa que $G$ é autocontraditório. Segue-se, então, um argumento que visa provar que a nulidade eterna da extensão de $[\lambda x(x \neq x)]$ é conseqüência do fato do zero ter sido definido como o número que pertence a tal conceito. Para que tal argumento seja levado a termo, é necessária a introdução do princípio (F5'), uma versão de (F5) que afirma que existe pelo menos um conceito autocontraditório ao qual o zero pertence:

(1) $\forall G\left[\left(G^{*} \wedge 0=\{F / F \approx G\}\right) \rightarrow \forall t \varepsilon G(t)=\varnothing\right]\left(F 1^{\prime}\right)$

(2) $\exists \chi\left(\chi^{*} \wedge 0=\{F / F \approx \chi\}\right)\left(F 5^{\prime}\right)$

(3) $[\lambda x(x \neq x)]^{*} \wedge 0=\{F / F \approx[\lambda x(x \neq x)]\}$ (2) Inst. Exist.

(4) $\left([\lambda x(x \neq x)]^{*} \wedge 0=\{F / F \approx[\lambda x(x \neq x)]\}\right) \rightarrow \forall t[\lambda x(x \neq x)](t)=\varnothing$ (1) Inst. Univ.

(5) $\forall t \varepsilon[\lambda x(x \neq x)](t)=\varnothing(3),(4)$ MP.

Conforme o argumento acima, a nulidade eterna da extensão do conceito "não ser idêntico a si mesmo" se explica porquanto tal conceito é autocontraditório e é usado para identificar o zero como o número que lhe pertence. Entretanto, tal argumento não especifica o que devemos entender por um conceito autocontraditório. Parece ser um tanto evidente que o se espera de um conceito desta natureza seja a sua incondicional incapacidade lógica de ser aplicado a algum objeto ou, em outras palavras, o que faz um conceito ser autocontraditório é o fato de sua extensão ser vazia em todos os mundos possíveis e em todos os instantes de tempo, isto é:

$$
\text { (F6) }[\lambda x \varphi x]^{*} \equiv_{\mathrm{df}} \forall t \forall w(\varepsilon[\lambda x \varphi x](t, w)=\varnothing)
$$

Vê-se que a definição de conceito autocontraditório nos diz que a extensão de tais conceitos é vazia em todos os instantes de tempo e em todos os mundos possíveis. De fato, para ser autocontraditório, não basta a um conceito ter uma extensão eternamente vazia. Por exemplo, o conceito 
"ser filho de Kant" tem extensão vazia em todos os instantes de tempo, mas não é autocontraditório, pois é concebível um mundo em que Kant tenha tido herdeiros. Além da "vaziez" eterna, a extensão de um conceito autocontraditório deve ser necessariamente nula. Isto implica que podemos eliminar a quantificação para os instantes de tempo e ficarmos somente com o quantificador para mundos possíveis, dentre os quais, obviamente, se situaria o mundo real.

Entretanto, o que intuitivamente se espera de um conceito autocontraditório é que, em todos os mundos possíveis, a nulidade de sua extensão se dê por conta de tal conceito violar explicitamente o princípio de não contradição, ou alguma outra lei fundamental da lógica. Mas tais leis da lógica são aplicáveis aos objetos em geral, enquanto estes se apresentam no tempo, seja este entendido como um todo contínuo, ou apenas um mero instante isolado. Isto é bem evidente, como nos diz Bolzano, em relação ao princípio de não contradição: não nos é possível conceber um objeto que, no instante $t$, tenha duas propriedades contraditórias. Se se permite a passagem do instante $t$ ao instante $t$ ', então é perfeitamente concebível que, em $t$, um certo objeto tenha a propriedade $P$ e que em $t$ ' não mais a tenha (o que implica que, em $t^{\prime}$, o objeto em questão tem a propriedade não- $P$ ). Mas a atribuição simultânea de $P$ e não- $P$ ao mesmo objeto é impensável, em qualquer mundo possível. Consideremos agora o princípio de identidade, outra lei fundamental da lógica. Em um determinado instante $t$, a descrição completa de todas as propriedades de um objeto nos dá a sua determinação como único. Chamemos tal descrição de $a(t)$. Em outro instante $t^{\prime}$, a determinação da unicidade do objeto pode mudar, posto que algumas das propriedades presentes em $a(t)$ podem não figurar em $a\left(t^{\prime}\right)$. Desta forma, o objeto em pauta mudou ao passar de $t$ para $t^{\prime}$, porquanto $a(t) \neq a\left(t^{\prime}\right)$. Mas é inadmissível que, para qualquer instante $t$, em qualquer mundo possível, tenhamos $a(t) \neq a(t)$. Desta maneira, a força coercitiva das leis da lógica pressupõe o tempo (mesmo que na qualidade de um único instante) como contexto básico em que se encontram os objetos; não se pode afirmar e negar algo simultaneamente de um mesmo objeto.

Portanto, quando se diz que os conceitos autocontraditórios têm extensão nula em todos os mundos possíveis, o que de fato está sendo dito é que a extensão de tais conceitos é nula em qualquer instante de tempo $t_{w}$ (mesmo que único) definido em $w$. Portanto, o que se espera de um conceito autocontraditório $[\lambda \times \varphi x]$ é o seguinte: 
(F7) $\varepsilon[\lambda x \varphi x]\left(t_{w}\right)=\varnothing$

Portanto, uma definição adequada de conceito autocontraditório é a seguinte:

$$
\text { (F8) }[\lambda x \varphi x]^{*} \equiv_{\mathrm{df}} \forall w \forall t_{w}\left(\varepsilon[\lambda x \varphi x]\left(t_{w}\right)=\varnothing\right)
$$

Como "ser diferente de si mesmo" é tomado por Frege como um conceito autocontraditório, então se segue que:

$$
\text { (F9) } \forall w \forall t_{w}\left(\varepsilon[\lambda x(x \neq x)]\left(t_{w}\right)=\varnothing\right)
$$

De posse de uma ontologia em que a validade incondicional do princípio de identidade - sob a forma de $\forall x(x=x)$ - é admitida, podemos inferir que o mundo real, assim como qualquer outro mundo possível, em qualquer estado considerado ou em qualquer instante de tempo, terá a totalidade de seus objetos excluída da extensão do conceito "ser diferente de si mesmo". Consideremos um instante real qualquer $t_{W}$, disposto em um continuum da totalidade dos instantes temporais. Por (F9), temos que:

$$
\text { (F10) } \varepsilon[\lambda x(x \neq x)]\left(t_{W}\right)=\varnothing \text {. }
$$

Em (F10), "W" representa o mundo real - aquele que tomamos como sendo a realidade tal qual esta se apresenta em um sentido físico ou objetivo. Mas não só isto: para qualquer $\Delta t$, tal que $|\Delta t|>\delta$ (sendo $\delta$ um número real tão pequeno quanto queiramos), sempre será o caso de:

$$
\text { (F9) } \varepsilon[\lambda x(x \neq x)]\left([t+\Delta t]_{W}\right)=\varnothing \text {. }
$$

A identidade acima nos diz que, saindo de $t$, podemos andar tanto para frente quanto para trás no continuum temporal (a eternidade), sem que isto altere a extensão do conceito $[\lambda x(x \neq x)]$; a extensão de tal conceito $e$ é uma função constante em relação a qualquer caminho que fizermos sobre a "eternidade". Embora outras extensões de conceito possam ser momentaneamente vazias, o conceito $[\lambda x(x \neq x)]$ define um objeto - a sua extensão - que é permanentemente o mesmo: o vazio eterno. 
Mas não só isto: a nulidade da extensão do conceito "ser diferente de si mesmo" é postulada na eternidade de todos os mundos possíveis. Em qualquer mundo possível, mesmo que neste mundo só haja um único instante (algo como o "eterno hoje", de Santo Agostinho), a extensão do conceito "ser diferente de si mesmo" é vazia em relação ao instante considerado. Não há como prescindir do tempo ou da instantaneidade pura na constatação de que "ser diferente de si mesmo" é autocontraditório; a sua extensão é sempre nula em qualquer mundo que se possa conceber. Por conseguinte, a razão última que leva a escolha dos conceitos autocontraditórios como adequados à definição lógica do zero é a intuição de que nada cai sob tais conceitos, em nenhum instante de tempo, seja este real ou possível.

Assim, se Frege admite que os números - em especial, o zero chega até nós sem o concurso da intuição espaço-temporal (como ele expressamente afirma em sua crítica ao dictum de Kant, como aqui foi apresentada), posto que definido somente com noções lógicas, o próprio Frege deveria concordar que tal definição lógica dos números, a partir do zero, se fundamenta no postulado de que há um objeto - a extensão de $[\lambda x(x \neq x)]-$ que é necessariamente eterno e vazio. Contudo, tal imutabilidade e "vaziez" necessárias em função do tempo, antes de caracterizar a atemporalidade de tal extensão de conceito - assim como de todas associadas a conceitos autocontraditórios -, nos diz que o caráter lógico do zero está ancorado na existência de extensões de conceito que estão no tempo, mas não mudam, assim como o Deus de Bolzano.

Evidentemente, a extensão de $[\lambda x(x \neq x)]$ é um objeto muito peculiar posto que se identifica com o eterna e necessariamente vazio - e, como tal, ao seu acesso pouca valia tem a sensibilidade, esta última entendida como receptáculo das impressões dos objetos cotidianos. Neste sentido, a crítica de Frege a Kant faz todo sentido: se o postulado de que o zero é um objeto lógico, abstrato, tem por fundamento a existência de um objeto que não muda com o tempo - a extensão do conceito "ser diferente de si mesmo"-, isto não implica que precisemos ter acesso imediato, via sensação, a este objeto constante no decorrer do fluxo temporal; na qualidade de necessariamente eterna, a extensão de $[\lambda x(x \neq x)]$, assim como a "substância divina", não se mostra de forma ordinária, corriqueira, como um mero objeto corruptível. Entretanto, a tese de que tais objetos imutáveis seriam atemporais, situados para além do espaço e do tempo, parece ser gratuita ou 
puramente arbitrária; ser eterno, como entendido por Bolzano e atestado implicitamente por Frege, com sua definição do zero, não é uma qualidade que se compreenda para além do tempo, mas, ao contrário, pressupõe o tempo como pano de fundo. Desta maneira, o platonismo fregeano, ao invés de descartar o tempo como um elemento estranho ao universo dos objetos lógicos, deve, preferencialmente, tomá-lo como a compleição inteira de todos os instantes reais ou possíveis, no decurso da qual alguns objetos lógicos - como a extensão de conceitos autocontraditórios - permanecem imutáveis. Por meio destes objetos privilegiados, por assim dizer, o caráter abstrato e eterno da aritmética faz-se legítimo. 


\section{Referências:}

BOLZANO, B. Paradoxien des Unendlichen, [1853]. Les Paradoxes de L'Infini. Introduction, Traduction de l'Allemand et Notes par Hourya Sinaceur. Ouvrages publié avec le concours du Centre National des Lettres. Paris: Éditions du Seuil, [1993].

."Versuch einer objectiven Begründung der Lehre von den drei

Dimensionen des Raumes", [1842]. In: Mathematischen-physikalische und philosophische Schriften 1842-1843. Herausgegeben von Gottfried Gabriel, Mathias Gatzmeier und Friedrich Kambartel. Friedrich Fromman Verlag. Günther Holzbog. Stuttgart - Bad Cannstatt, [1989];

BROUWER, L. E. J. "Consciouness, Philosophy, and Mathematics". Proceedings of the Tenth International Congress of Philosophy. Amsterdam, North Holland, [1949]; FREGE, G. Begriffsschrift, einer arithmetischen nachgebildete Formelsprache des reinen Densken, [1879]. Conceptual Notation and Related Articles. Translated and edited by T.W. Bynum, Oxford: Clarendon Press, [1972];

. Grundlagen der Arithmetik. Eine logisch mathematische Untersuchung über den Begriff der Zahl [1884]. Foundations of Arithmetic. A logico-mathenatical Enquiry into the Concept of Number. English translation by J. L. Austin, M. A. Second Revised Edition. Basil Blackwell Oxford, [1978];

.Grungesetze der Arithmetik, Vol I. Jena: H. Pohle. Translated in part by M. Furth in The Basic Laws ofArithmetc: Exposition of the System. Los Angeles: University of California Press.

KANT, I. Kritik der reinen Vernunft, [1781]. Crítica da Razão Pura. Tradução de Manuela Pinto dos Santos e Alexandre Fradique Mourão. Introdução e notas de Alexandre Fradique Mourão. Segunda Edição. Fundação Calouste Gulbenkian, Lisboa, [1966?].

ZALTA, E. An Introduction to Axiomatic Metaphysics. Dordrecht/ Boston/ Lancaster: D. Reidel Publishing Company, [1981].

Email: waltergomide@yahoo.com 